\title{
AN APPLICATION OF A THEOREM OF SINGER
}

\author{
by D. M. CONNOLLY and J. H. WILLIAMSON
}

(Received 22nd March 1973)

The authors have recently treated (2) the problem of finding subsets $E$ of the real line $R$, of type $F_{\sigma}$, such that $E-E$ contains an interval and the $k$-fold vector sum $(k) E$ is of measure zero. Positive results can be obtained, for all $k$, on the basis of a recent theorem of J. A. Haight (3), following earlier partial results (1), (4) for $k \leqq 7$; and indeed in these cases the problem has a solution with $E$ a perfect set. An analogous problem, apparently in most respects subtler than the first, is the following. Do there exist finite regular Borel measures $\mu$ on $\mathbb{R}$ such that $\mu * \tilde{\mu}$ is absolutely continuous (where $\tilde{\mu}$ is the adjoint of $\mu$ ) and the $k$ th convolution power $\mu^{k}$ is singular? Both problems are of interest in the general context of elucidating the properties of the measure algebra $M(R)$ or, more generally, $M(G)$ for locally compact abelian $G$. The second problem may be regarded as an attempt to provide (at least one aspect of) a multiplicity theory for the first.

The purpose of the present note is to point out that the necessary machinery for a positive solution of this second problem in the simplest case, $k=2$, has been available for many years, in a classical theorem of Singer (7, pp. 380-381; see also 6, Theorem 6.1); we give the solution in this case. It appears that the construction has inherent limitations and necessarily fails to provide a solution for large $k$. We show by an elementary argument that it must break down for $k \geqq 6$. This does not, of course, imply that the result is false in such cases. It would seem that for $k \geqq 3$ a subtler approach than ours is needed to determine the truth of the matter.

We begin with some considerations involving measures on $\mathbb{R}$. We shall produce measures with the required properties by taking suitable infinite convolution products of simple probability measures. We shall say that a sequence $v_{n}$ of measures tends weakly to a limit $v$ if $v_{n}(I) \rightarrow v(I)$ for each interval of continuity of $v$ (i.e. $I$ is to be open and its end-points are not atoms for $v$ ). In the present context this mode of convergence has various equivalent formulations.

Lemma 1. Let $\left(v_{n}\right)$ be a sequence of non-negative measures on $\mathbb{R}$ with the properties

(i) $v_{n} \rightarrow v$ weakly,

(ii) there is a constant $c>0$ such that for any interval I, of length $l(I)$,

$$
\limsup _{n \rightarrow \infty} v_{n}(I) \leqq c l(I) \text {. }
$$


Then $v$ is absolutely continuous: in fact

where $0 \leqq f(x) \leqq c$ for all $x$.

$$
v(A)=\int_{A} f(x) d x
$$

Proof. This is a straightforward verification.

Suppose next that $q=\left(q_{1}, q_{2}, \ldots,\right)$ is a sequence of integers with $q_{r} \geqq 2$ for all $r \in \mathbb{N}$. Write $d_{r}=\left(q_{1} q_{2} \ldots q_{r}\right)^{-1}$. For each $r \in \mathbb{N}$ let $F_{r}$ be a set of integers, $F_{r}=\left\{n_{1}^{(r)}, \ldots, n_{\sigma_{r}}^{(r)}\right\}$, say. Let $\mu_{r}$ be a probability measure (that is, non-negative and of total mass 1) supported on the points $n^{(r)} d_{r}\left(n^{(r)} \in F_{r}\right)$. We shall suppose, to simplify the situation, that each $n^{(r)} \in F_{r}$ is essential, that is, $\mu_{r}\left(n^{(r)} d_{r}\right)>0$ for each $n^{(r)}$. It is not always true that $\mu=\mu_{1} * \mu_{2} * \ldots$ converges; and even if it does its total mass may be strictly less than 1 . However, if there is a constant $C$, independent of $r$, with $\left|n^{(r)}\right| \leqq C q_{r}$ for all $n^{(r)} \in F_{r}$ and all $r$

then $\mu_{1} * \mu_{2} \ldots$ converges weakly to a probability measure $\mu$. The support of $\mu$ is the set of all points that admit a development of the form $\sum_{r=1}^{\infty} n^{(r)} d_{r}$, with $n^{(r)} \in F_{r}$. Condition (1) is satisfied in all the cases with which we are concerned.

Lemma 2. Suppose that (1) holds, and also the residues of the $n^{(r)} \bmod q_{r}$ form a complete set.

Let $\mu_{r}$ be a probability measure on the points $n^{(r)} d_{r}$ such that for each integer $a$, $0 \leqq a \leqq q_{r}-1$, the total mass of $\mu_{r}$ on the $n^{(r)} d_{r}$ with $n^{(r)} \equiv$ a mod $q_{r}$ is exactly $q_{r}^{-1}$. Then $\mu=\mu_{1} * \mu_{2} * \ldots$ is absolutely continuous.

Proof. Write $v_{r}=\mu_{1} * \mu_{2} * \ldots * \mu_{r}$. Let $E_{r}$ be the set of numbers of the form $\sum_{s=1}^{r} n^{(s)} d_{s}$, with $n^{(s)} \in F_{s}(1 \leqq s \leqq r)$ and $E$ the set of all numbers of the form $\sum_{s=1}^{\infty} n^{(s)} d_{s}$ with $n^{(s)} \in F_{s}(s \geqq 1)$. The measure $v_{r}$ is concentrated on $E_{r}$, and the total mass concentrated on any one point of $E_{r}$ does not exceed $d_{r}$, as is easily verified. Moreover, the points of $E_{r}$ are distant at least $d_{r}$ from each other. The measures $v_{r}$ converge weakly to a limit $\mu$, whose support is $E$, and it is immediate from Lemma 1 that $\mu$ is absolutely continuous (with $c=1$ ).

Lemma 3. If (1) and (2) hold, and if $\mu_{r}$ is a probability measure on the $n^{(r)} d_{r}$ with $\mu_{r}=\mu_{r}^{\prime}+\mu_{r}^{\prime \prime}$, where $\mu_{r}^{\prime}$ satisfies the conditions specified for $\mu_{r}$ in Lemma 2, and

$$
\sum_{r=1}^{\infty}\left\|\mu_{r}^{\prime \prime}\right\|<\infty
$$

then $\mu=\mu_{1} * \mu_{2} * \ldots$ is absolutely continuous.

Proof. It is clear from Lemma 2 that $\mu^{\prime}=\mu_{1}^{\prime} * \mu_{2}^{\prime} * \ldots$ is absolutely continuous; 
the present Lemma is now a consequence of the classical theorem of Jessen and Wintner (5, Theorem 35).

The explicit reference to Jessen and Wintner's theorem can be avoided in the present case by a simple direct argument. For each $r \in \mathbb{N}$ we can write

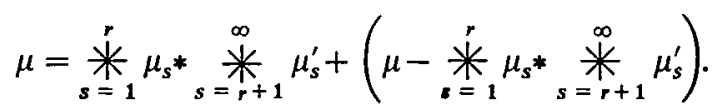

The first term is clearly absolutely continuous and the norm of the second is $1-\prod_{s=r+1}^{\infty}\left(1-\left\|\mu_{s}^{\prime \prime}\right\|\right)$; this $\rightarrow 0$ as $r \rightarrow \infty$. The measure $\mu$ is then the limit in norm of absolutely continuous measures, and is thus itself absolutely continuous.

Lemma 4. If (1) holds, and now $F_{r}=\left\{n_{1}^{(r)}, \ldots, n_{\sigma_{r}}^{(r)}\right\}$ is a set of integers with

$$
\sigma_{r} \leqq\left(1-\omega_{r}\right) q_{r} \text {, where } \prod_{r=1}^{\infty}\left(1-\omega_{r}\right)=0
$$

and $\mu_{r}$ is a probability measure on the points $n^{(r)} d_{r}$ then $\mu=\mu_{1} * \mu_{2} * \ldots$ is singular. most

Proof. If as before $v_{r}=\mu_{1} * \mu_{2} * \ldots * \mu_{r}$ then the support $E_{r}$ of $v_{r}$ contains at

$$
d_{r}^{-1} \prod_{s=1}^{r}\left(1-\omega_{s}\right)
$$

points, all of the form $n d_{r}$. For any given $a, b$ the proportion of the points of the form $n d_{r}$ that are in $\mathrm{E}_{\boldsymbol{r}} \cap[a, b]$, as compared with $[a, b]$, tends to 0 as $r \rightarrow \infty$ because $\Pi\left(1-\omega_{s}\right)$ diverges to 0 . It follows that the support of $\mu=\lim v_{r}$ is of measure 0 , and this in turn implies that $\mu$ is singular ( $\mu=0$ is excluded by condition (1)).

We now come to the main construction. Let $a_{r}$ be any prime-power; Singer has established (7, pp. 380-381) the existence of a set $A_{r}$ of $a_{r}+1$ residues mod $a_{r}^{2}+a_{r}+1$, such that $A_{r}-A_{r}$ contains all residues, with zero represented $a_{r}+1$ times and the other $a_{r}^{2}+a_{r}$ residues once each. We may suppose without loss of generality that the elements of $A_{r}$ are integers $n$ with $0 \leqq n<a_{\mathrm{r}}^{2}+a_{\mathrm{r}}+1$. Let $q_{r}=a_{r}^{2}+a_{r}+1$, and let $\lambda_{r}$ be the measure with mass $\left(a_{r}+1\right)^{-1}$ at each point $n d_{r},\left(n \in A_{r}\right)$.

Theorem 1. If $\sum_{r=1}^{\infty} a_{r}^{-1}<\infty$ then $\lambda=\lambda_{1} * \lambda_{2} * \ldots$ is such that $\lambda * \tilde{\lambda}$ is absolutely continuous and $\lambda^{2}$ is singular.

Proof. Write $\mu_{r}=\lambda_{r} * \tilde{\lambda}_{r}$ : then it is immediate that $\mu_{r}$ has mass $\left(a_{r}+1\right)^{-1}$ at 0 and $\left(a_{r}+1\right)^{-2}$ at the other $a_{r}^{2}+a_{r}$ points of $\left\{n d_{r}: n \in A_{r}-A_{r}\right\}$. Let $\mu_{r}^{\prime}$ have mass $\left(a_{r}^{2}+a_{r}+1\right)^{-1}$ at each point of $\left\{n d_{r}: n \in A_{r}-A_{r}\right\}$; if $\mu_{r}=\mu_{r}^{\prime}+\mu_{r}^{\prime \prime}$ then it is clear that

$$
\left\|\mu^{\prime \prime}\right\|=2 a_{r}^{2} /\left(a_{r}+1\right)\left(a_{r}^{2}+a_{r}+1\right) \sim 2 a_{r}^{-1}
$$

E.M.S.-19/2-I 
and we are thus in the situation of Lemma 3. It follows from that lemma that $\mu=\mu_{1} * \mu_{2} * \ldots=\lambda_{1} * \tilde{\lambda}_{1} * \lambda_{2} * \tilde{\lambda}_{2} * \ldots=\lambda * \tilde{\lambda}$ is absolutely continuous.

Moreover, if $v_{r}=\lambda_{r}^{2}$, the support of $v_{r}$ is $\left\{n d_{r}: n \in(2) A_{r}\right\}$, and this contains at most $a_{r}+1+\frac{1}{2}\left(a_{r}^{2}+a_{r}\right)$ points. With $q_{r}=a_{r}^{2}+a_{r}+1$ this leads to

$$
\lim \sup \left(1-\omega_{r}\right) \leqq \frac{1}{2}
$$

and hence $\Pi\left(1-\omega_{r}\right)=0$. It follows from Lemma 4 that

is singular.

$$
v=v_{1} * v_{2} * \ldots=\lambda_{1} * \lambda_{1} * \lambda_{2} * \lambda_{2} * \ldots=\lambda^{2}
$$

One consequence of Theorem 1 is the following:

Corollary. There exists on $\mathbb{R}$ a function $f$ (necessarily continuous and tending to zero at $\pm \infty$ ) that is the Fourier-Stieltjes transform of a singular measure, such that $|f|$ is the Fourier-Stieltjes transform of an absolutely continuous measure.

Proof. Let $\lambda$ be as in Theorem 1 and let $f$ be the Fourier-Stieltjes transform of $\lambda^{2}$.

We conclude by showing, as promised, that our method does not seem well suited to discuss the cases of larger values of $k$.

Let $M(\mathbb{R}, d)$ be the subset (in fact a subalgebra) of $M(\mathbb{R})$ consisting of measures supported on the subgroup $\{n d: n \in \mathbb{Z}\}$, and let $q$ be a positive integer. Defining the map $\phi$ from $M(\mathbb{R}, d)$ to $M(\mathbb{Z}(q))$ by $(\phi \mu)(a)=\sum_{n \equiv a \bmod q} \mu(n d)$ it is clear that $\phi$ is a homomorphism. In general $\|\phi \mu\| \leqq\|\mu\|$, and if for each $a$ all the $\mu(n d)$ with $n \equiv a \bmod q$ have the same sign (or the same phase in the complex case) then $\|\phi \mu\|=\|\mu\|$. It will be convenient in the following to look at the images under $\phi$ of measures on $\mathbb{R}$ rather than directly at the measures themselves.

Theorem 2. If $\lambda$ is as in Theorem 1 then $\lambda^{6}$ is absolutely continuous.

Proof. Let $q_{r}, d_{r}$ be as before and write $G=\mathbb{Z}\left(q_{r}\right)$. Let $\phi$ be defined as above relative to $d_{r}, q_{r}$. Let $\phi \lambda_{r}=\alpha_{r}$; then $\alpha_{r} * \tilde{\alpha}_{r}=\beta_{r}+\gamma_{r}$, say with

$$
\beta_{r}=\phi \mu_{r}^{\prime}, \gamma_{r}=\phi \mu_{r}^{\prime \prime} \text {. }
$$

It is clear that $\beta_{r}$ is Haar measure on $G$ (normalised to have mass 1 ), and that $\left\|\gamma_{r}\right\|=\left\|\mu_{r}^{\prime \prime}\right\|=2 a_{r}^{2} /\left(a_{r}+1\right)\left(a_{r}^{2}+a_{r}+1\right)$. Taking Fourier transforms, defined for any $\sigma \in M(G)$ by $\hat{\sigma}(x)=\sum_{y \in G} \sigma(y)\langle y, x\rangle$, so that

$$
\sigma(x)=\frac{1}{|G|} \sum_{y \in G} \overline{\hat{\sigma}(y)\langle x, y\rangle}
$$

where $\hat{G}(\cong G$, here) is the dual group of $G$, we have

$$
\left|\hat{\alpha}_{r}(x)\right|^{2}=\hat{\beta}_{r}(x)+\hat{\gamma}_{r}(x)
$$

where $\hat{\beta}_{r}(0)=1, \hat{\beta}_{r}(x)=0$ if $x \neq 0$ and (since $\left.\hat{\alpha}_{r}(0)=1\right) \hat{\gamma}_{r}(0)=0,\left|\hat{\gamma}_{r}(x)\right| \leqq\left\|\gamma_{r}\right\|$ everywhere. 
We may thus write

$$
\hat{\alpha}_{r}(x)=\hat{\beta}_{r}(x)+\hat{\varepsilon}_{r}(x),
$$

where $\hat{\varepsilon}_{r}(0)=0$ and $\left|\hat{\varepsilon}_{r}(x)\right| \leqq\left\|\gamma_{r}\right\|^{\frac{1}{2}}$ everywhere, and it follows that for any $k \in \mathbb{N}$ we have

$$
\hat{\alpha}_{r}^{k}(x)=\dot{\beta}_{r}(x)+\hat{\varepsilon}_{r}^{k}(x)
$$

Thus $\left|\hat{\varepsilon}_{k}^{r}(x)\right| \leqq\left\|\gamma_{r}\right\|^{k / 2}$ everywhere, whence $\left|\varepsilon_{r}^{k}(y)\right| \leqq \frac{1}{|G|}|G|\left\|\gamma_{r}\right\|^{k / 2}$, and $\left\|\varepsilon_{r}^{k}\right\|=\sum_{y \in G}\left|\varepsilon_{r}^{k}(y)\right| \leqq|G|\left\|\gamma_{r}\right\|^{k / 2}$

$$
=\left(a_{r}^{2}+a_{r}+1\right)\left[2 a_{r}^{2} /\left(a_{r}+1\right)\left(a_{r}^{2}+a_{r}+1\right)\right]^{k / 2} \sim 2^{k / 2} a_{r}^{2-k / 2} .
$$

Let $\theta_{r}$ be chosen so that $\phi \theta_{r}=\varepsilon_{r}^{k},\left\|\theta_{r}\right\|=\left\|\varepsilon_{r}^{k}\right\|$, and $\lambda_{r}^{k}-\theta_{r}$ is non-negative. Then $\phi\left(\lambda_{r}^{k}-\theta_{r}\right)$ is Haar measure on $G$, so $\lambda_{r}^{k}-\theta_{r}$ satisfies the requirement for $\mu_{r}^{\prime}$ in Lemma 3, while $\left\|\theta_{r}\right\|=\left\|\varepsilon_{r}^{k}\right\| \sim 2^{k / 2} a_{r}^{2-k / 2}$. Thus, if $k \geqq 6, \Sigma\left\|\theta_{r}\right\|<\infty$ (since $\left.\Sigma a_{r}^{-1}<\infty\right)$ and all the conditions of Lemma 3 hold. Thus

is absolutely continuous.

$$
\underset{r=1}{*} \lambda_{r}^{6}=\left(\underset{r=1}{\stackrel{\infty}{*} \lambda_{r}}\right)^{6}=\lambda^{6}
$$

It is evident that if $a_{r} \rightarrow \infty$ more rapidly, so that $\Sigma a_{r}^{-\frac{1}{2}}<\infty$, then $\lambda^{5}$ is already absolutely continuous. It seems likely that these results could be substantially improved by the use of more delicate arguments.

\title{
REFERENCES
}

(1) D. M. Connolly, Integer difference-covers which are not $k$-sum-covers, for $k=6,7$, Proc. Cambridge Philos. Soc. 74 (1973), 17-28.

(2) D. M. Connolly and J. H. Williamson, Difference-covers that are not $k$-sum-covers II, Proc. Cambridge Philos. Soc. 75 (1974), 63-73.

(3) J. A. HaIght, Difference-covers which have small $k$-sums for any $k$, Mathematika 20 (1973), 109-118.

(4) T. H. JACKson, J. H. Williamson and D. R. Woodall, Difference-covers that are not $k$-sum covers I, Proc. Cambridge Philos. Soc. 72 (1972), 425-438.

(5) B. Jessen and A. Wintner, Distribution functions and the Riemann zetafunction, Trans. Amer. Math. Soc. 38 (1935), 48-88.

(6) H. B. MANN, Addition Theorems (Interscience tracts in pure and applied mathematics no. 18, Interscience Publishers, New York, 1965).

(7) J. Singer, A theorem in finite projective geometry and some applications to number theory, Trans. Amer. Math. Soc. 43 (1938), 377-385.

\author{
UNIVERSITY OF LETHBRIDGE \\ CANADA \\ and \\ UNIVERSITY OF YORK \\ ENGLAND
}

